\title{
ASYMPTOTIC BEHAVIOR OF FUNCTIONS WITH BOUNDED BOUNDARY ROTATION
}

\author{
BY \\ JAMES W. NOONAN( ${ }^{(1)}$
}

\begin{abstract}
For $k \geqq 2$ denote by $V_{k}$ the class of normalized functions, analytic in the unit disc, which have boundary rotation at most $k \pi$. Let $a_{n}$ be the $n$th Taylor coefficient of $f(z) \in V_{k}$. Let $I_{\lambda}\left(r, f^{\prime}\right)$ and $I_{\lambda}(r, f)$ be the $\lambda$-integral mean of $f^{\prime}(z)$ and $f(z)$ respectively. We determine asymptotic formulas for $f^{\prime}(z)$, and these formulas are then applied to study the behavior of $\left|a_{n}\right|$ as $n \rightarrow \infty$, and the behavior of $I_{\lambda}\left(r, f^{\prime}\right)$ and $I_{\lambda}(r, f)$ as $r \rightarrow 1$.
\end{abstract}

1. Introduction. Let us denote by $V_{k}$ the set of all functions $f(z)$ analytic in $U=\{z:|z|<1\}$ such that $f(0)=0$ and

$$
f^{\prime}(z)=\exp \left\{\frac{1}{\pi} \int_{0}^{2 \pi} \log \left(1-z e^{-i t}\right)^{-1} d \mu(t)\right\}
$$

where $\mu(t)$ is a real-valued function of bounded variation on $[0,2 \pi]$ satisfying

$$
\int_{0}^{2 \pi} d \mu(t)=2 \pi, \quad \int_{0}^{2 \pi}|d \mu(t)| \leqq k \pi .
$$

$V_{k}$ is the class of functions with boundary rotation at most $k \pi$. It is clear that $k \geqq 2$, and when $k=2, V_{k}$ is the class of normalized convex functions. It is also known [10] that, for $2 \leqq k \leqq 4, V_{k}$ contains only schlicht functions.

If $f(z)=z+a_{2} z^{2}+\cdots$, consider the problem $A_{n}(k)=\max \left\{\left|a_{n}\right|: f \in V_{k}\right\}$. This problem has been solved for all $n$ only when $k=2$ [8] and when $k=4$ [13]. Also, the problem has been solved for all $k \geqq 2$ only when $n=2$ [6], $n=3$ [6], and $n=4$ [2]. In all the above cases, the extremal function has been

$$
F(z)=\frac{1}{k}\left\{\left(\frac{1+z}{1-z}\right)^{k / 2}-1\right\}=z+\sum_{n=2}^{\infty} A_{n} z^{n} .
$$

The purpose of this paper is to examine the asymptotic behavior of $V_{k}$ functions. We shall derive asymptotic formulas for $f^{\prime}(z)$ which show that for any fixed $f(z)$

Received by the editors February 1, 1971.

AMS 1970 subject classifications. Primary 30A34, 30A32; Secondary 30A40.

Key words and phrases. Asymptotic behavior, bounded boundary rotation, convex functions, starlike functions, coefficients, integral mean.

( ${ }^{1}$ These results are part of the author's Ph.D. dissertation, written at the University of Maryland under the direction of Professor W. E. Kirwan. The author at present holds a National Research Council Postdoctoral Resident Research Associateship supported by the Naval Research Laboratory, Washington, D. C.

Copyright (1) 1972, American Mathematical Society 
$=z+a_{2} z^{2}+\cdots \in V_{k}$, there exists a positive integer $n_{0}(f)$ depending on $f(z)$ such that $n \geqq n_{0}(f)$ implies $\left|a_{n}\right| \leqq A_{n}$, where $A_{n}$ is as in (1.3). These same formulas will also be used to study the behavior of the integral means

$$
\begin{gathered}
I_{\lambda}\left(r, f^{\prime}\right)=\frac{1}{2 \pi} \int_{0}^{2 \pi}\left|f^{\prime}\left(r e^{i \theta}\right)\right|^{\lambda} d \theta, \\
I_{\lambda}(r, f)=\frac{1}{2 \pi} \int_{0}^{2 \pi}\left|f\left(r e^{i \theta}\right)\right|^{\lambda} d \theta .
\end{gathered}
$$

The method used to derive these asymptotic formulas is due originally to Littlewood [7, pp. 93-95], and it was later generalized by Hayman [3, pp. 106-108]. Our method is that of Hayman, although the proofs are different because we have no assumption on the mean-valency of $f(z) \in V_{k}$.

2. Notation and background material. In order to insure uniqueness (up to additive constants) of the integrator $\mu(t)$ in (1.1), we shall require that $\mu(t)$ be normalized in the sense that $\mu(t)=(\mu(t+0)+\mu(t-0)) / 2$, where at $t=0, t=2 \pi$ we extend $\mu(t)$ periodically before normalizing. We shall also write $\mu(t)=\nu(t)-\sigma(t)$, where $\nu(t)=\left(\mu(t)+\mathrm{V}_{0}^{t}(\mu)\right) / 2, \sigma(t)=\left(\mathrm{V}_{0}^{t}(\mu)-\mu(t)\right) / 2$, and where $\mathrm{V}_{0}^{t}(\mu)$ is the total variation of $\mu$ from 0 to $t$. In addition we write $\alpha(\theta)=\nu(\theta+0)-\nu(\theta-0), \beta(\theta)$ $=\sigma(\theta+0)-\sigma(\theta-0)$, with the usual modifications at $\theta=0,2 \pi$. Since $\mu(t)$ is normalized, it follows that $\alpha(\theta)>0$ implies $\beta(\theta)=0$.

Let $\alpha=\max _{\theta} \alpha(\theta)$, and let $M\left(r, f^{\prime}\right)=\max \left\{\left|f^{\prime}(z)\right|:|z|=r\right\}$. Then as $r \rightarrow 1$ we have that

$$
\begin{aligned}
& \left(\log M\left(r, f^{\prime}\right)\right) /(\log 1 /(1-r)) \rightarrow \alpha, \\
& \left(\log \left|f^{\prime}\left(r e^{i \theta}\right)\right|\right) /(\log 1 /(1-r)) \rightarrow \alpha(\theta)-\beta(\theta) .
\end{aligned}
$$

The proofs of these facts are similar to the proof of Theorem 1 in [12], and complete details may be found in [9, pp. 15-21].

3. Asymptotic formulas for the coefficients. Following Hayman [3, p. 100] we first prove a regularity theorem.

THEOREM 3.1. Let $f(z) \in V_{k}$ with $\mu(t)=\nu(t)-\sigma(t)$ as its integrator.

Then the limit $\omega=\lim _{r \rightarrow 1}(1-r)^{k / 2+1} M\left(r, f^{\prime}\right)$ exists finitely, and $\omega=0$ unless $\nu(t)$ is a step function with a single jump of height $(k / 2+1) \pi$. In this case

$$
\omega=\lim _{r \rightarrow 1}(1-r)^{k / 2+1}\left|f^{\prime}\left(r \exp \left(i \theta_{0}\right)\right)\right|
$$

where $\nu\left(\theta_{0}+0\right)-\nu\left(\theta_{0}-0\right)=(k / 2+1) \pi$.

Proof. Since $f^{\prime}(z) \neq 0$ in $U, \log f^{\prime}(z)$ is analytic. Let $\theta$ be fixed but arbitrary, and let

$$
u(r)+i v(r)=\frac{\partial}{\partial r} \log f^{\prime}\left(r e^{i \theta}\right)=\frac{e^{i \theta} f^{\prime \prime}\left(r e^{i \theta}\right)}{f^{\prime}\left(r e^{i \theta}\right)}
$$


By a result of Robertson [14, Theorem 1]

$$
\left|\frac{f^{\prime \prime}(z)}{f^{\prime}(z)}\right| \leqq \frac{2|z|+k}{1-|z|^{2}}<\frac{2+k}{1-|z|^{2}}
$$

Thus $u^{2}(r)+v^{2}(r) \leqq\left((2+k) /\left(1-r^{2}\right)\right)^{2}$. Therefore

$$
\frac{\partial}{\partial r} \log \left|f^{\prime}\left(r e^{i \theta}\right)\right|=u(r) \leqq|u(r)| \leqq \frac{2+k}{1-r^{2}} .
$$

By taking $r_{1}<r_{2}$ and integrating, we find

$$
\log \left\{\left|f^{\prime}\left(r_{2} e^{i \theta}\right)\right|\left(\frac{1-r_{2}}{1+r_{2}}\right)^{k / 2+1}\right\} \leqq \log \left\{\left|f^{\prime}\left(r_{1} e^{i \theta}\right)\right|\left(\frac{1-r_{1}}{1+r_{1}}\right)^{k / 2+1}\right\} .
$$

By choosing $\theta$ such that $\left|f^{\prime}\left(r_{2} e^{i \theta}\right)\right|=M\left(r_{2}, f^{\prime}\right)$, we see that

$$
M\left(r, f^{\prime}\right)((1-r) /(1+r))^{k / 2+1}
$$

is a decreasing function of $r$, and thus approaches a limit as $r \rightarrow 1$. Therefore $\omega$ exists and is finite.

Since (1.2) implies $\int_{0}^{2 \pi} d \nu(t) \leqq(k / 2+1) \pi$, it follows directly from (2.1) that $\omega=0$ unless $\nu(t)$ is as claimed in the theorem. Suppose now that $\omega>0$ and let $\nu\left(\theta_{0}+0\right)$ $-\nu\left(\theta_{0}-0\right)=(k / 2+1) \pi$. Let $r_{n}=1-(1 / n)$ and choose $\theta_{n}$ such that $M\left(r_{n}, f^{\prime}\right)$ $=\left|f^{\prime}\left(r_{n} \exp \left(i \theta_{n}\right)\right)\right|$. Then $\left(1-r_{n}\right)^{k / 2+1}\left|f^{\prime}\left(r_{n} \exp \left(i \theta_{n}\right)\right)\right| \rightarrow \omega$. A simple application of (2.2) shows that $\theta_{n} \rightarrow \theta_{0}$ as $n \rightarrow \infty$. By (3.1) we have for $r \leqq r_{n}$ that

(3.2) $\left(1-r_{n}\right)^{k / 2+1}\left|f^{\prime}\left(r_{n} \exp \left(i \theta_{n}\right)\right)\right| \leqq(1-r)^{k / 2+1}\left|f^{\prime}\left(r \exp \left(i \theta_{n}\right)\right)\right|\left(\left(1+r_{n}\right) /(1+r)\right)^{k / 2+1}$.

Now let $r$ be fixed and let $n \rightarrow+\infty$ in (3.2). We see

$$
\omega \leqq(1-r)^{k / 2+1}\left|f^{\prime}\left(r \exp \left(i \theta_{0}\right)\right)\right|(2 /(1+r))^{k / 2+1} .
$$

Now letting $r \rightarrow 1$, we see that

$$
\omega \leqq \lim _{r \rightarrow 1} \inf (1-r)^{k / 2+1}\left|f^{\prime}\left(r \exp \left(i \theta_{0}\right)\right)\right| .
$$

But by definition of $\omega$ we have

$$
\lim _{r \rightarrow 1} \sup (1-r)^{k / 2+1}\left|f^{\prime}\left(r \exp \left(i \theta_{0}\right)\right)\right| \leqq \omega,
$$

so the theorem is proved.

Corollary 3.2. Let $f(z) \in V_{k}$. Then $M\left(r, f^{\prime}\right)((1-r) /(1+r))^{k / 2+1}$ is a decreasing function of $r$.

Before we study the behavior of $a_{n}$ as $n \rightarrow \infty$, we need two technical lemmas.

LEMMA 3.1. Let $f(z) \in V_{k}$ with $\omega=\lim _{r \rightarrow 1}(1-r)^{k / 2+1}\left|f^{\prime}\left(r \exp \left(i \theta_{0}\right)\right)\right|>0$. Let $\delta>0$ be given. Then we may choose $C(\delta)>0$ and $r(\delta)<1$ such that $r \geqq r(\delta)$ implies

$$
\frac{1}{2 \pi} \int_{y}\left|f^{\prime}\left(r e^{i \theta}\right)\right| d \theta<\frac{\delta}{(1-r)^{k / 2}}
$$

where $\gamma=\left\{\theta:(1-r) C(\delta) \leqq\left|\theta-\theta_{0}\right| \leqq \pi\right\}$. 
Proof. Without loss of generality we may assume $\theta_{0}=0$. From (1.1) (see also Theorem 3.1 in [1]) it follows that

$$
f^{\prime}(z)=\frac{\left(s_{1}(z) / z\right)^{(k+2) / 4}}{\left(s_{2}(z) / z\right)^{(k-2) / 4}}
$$

where $s_{1}(z)$ and $s_{2}(z)$ are normalized starlike functions. But $\omega>0$ and $\theta_{0}=0$ imply $s_{1}(z)=z /(1-z)^{2}$. Therefore

$$
\left|s_{1}(z) / z\right|=\left((1-r)^{2}+4 r \sin ^{2}(\theta / 2)\right)^{-1}<\pi^{2} / \theta^{2}
$$

for $r \geqq 1 / 4$ and for all $\theta \neq 0$. By using well-known distortion theorems to bound $\left|z / s_{2}(z)\right|$, we have

for $r \geqq 1 / 4$ and $\theta \neq 0$.

$$
\left|f^{\prime}\left(r e^{i \theta}\right)\right| \leqq(1+r)^{k / 2-1}\left(\pi^{2} / \theta^{2}\right)^{(k+2) / 4}
$$

Let $C$ be any positive constant, and let $\gamma=\{\theta:(1-r) C \leqq|\theta| \leqq \pi\}$. Then for $r$ sufficiently close to 1 we have

$$
\begin{aligned}
\frac{1}{2 \pi} \int_{\gamma}\left|f^{\prime}\left(r e^{i \theta}\right)\right| d \theta & \leqq \frac{2^{k / 2-2}}{\pi}\left\{\int_{(1-r) C}^{\pi}+\int_{-\pi}^{-(1-r) C}\right\}\left(\frac{\pi^{2}}{\theta^{2}}\right)^{(k+2) / 4} d \theta \\
& \leqq \frac{2^{k / 2} \pi^{k / 2}}{k} \frac{1}{C^{k / 2}(1-r)^{k / 2}} .
\end{aligned}
$$

Letting $C=C(\delta)=2 \pi /(k \delta)^{2 / k}$, we obtain the lemma.

For notational purposes let $\omega(r)=(1-r)^{k / 2+1} f^{\prime}\left(r \exp \left(i \theta_{0}\right)\right)$ where $\theta_{0}$ is as above. We then have

LEMMA 3.2. Let $f(z) \in V_{k}$ and let $\omega=\lim _{r \rightarrow 1}(1-r)^{k / 2+1}\left|f^{\prime}\left(r \exp \left(i \theta_{0}\right)\right)\right|>0$. Let $r_{n} \uparrow 1$ and let $f_{n}^{\prime}(z)=\omega\left(r_{n}\right) /\left(1-z \exp \left(-i \theta_{0}\right)\right)^{k / 2+1}$. Let $S$ be a fixed but arbitrary Stolz angle with vertex at $\exp \left(i \theta_{0}\right)$, and let $D_{n}=\left\{z \in S:\left|\exp \left(i \theta_{0}\right)-z\right|<2 / n\right\}$. Then $f_{n}^{\prime}(z) \sim f^{\prime}(z)$ as $n \rightarrow \infty$, uniformly for $z \in D_{n}$.

Proof. We again assume $\theta_{0}=0$. As above we have (3.3) with $s_{1}(z)=z /(1-z)^{2}$. Thus

$$
f_{n}^{\prime}(z)=\frac{1}{(1-z)^{k / 2+1}}\left\{\frac{r_{n}}{s_{2}\left(r_{n}\right)}\right\}^{(k-2) / 4}, \quad f^{\prime}(z)=\frac{1}{(1-z)^{k / 2+1}}\left\{\frac{z}{s_{2}(z)}\right\}^{(k-2) / 4} .
$$

To prove the lemma it clearly suffices to prove $s_{2}(z) / s_{2}\left(r_{n}\right) \rightarrow 1$ as $n \rightarrow \infty$, uniformly for $z \in D_{n}$.

We see that $\omega=\lim _{r \rightarrow 1}\left|r / s_{2}(r)\right|^{(k-2) / 4}$, so $0<\lim _{r \rightarrow 1}\left|s_{2}(r)\right|<\infty$. We also know [12, Lemma 1] $\lim _{r \rightarrow 1} \arg s_{2}(r)$ exists. Thus $L=\lim _{r \rightarrow 1} s_{2}(r)$ exists. Let $S_{1}$ be a Stolz angle properly containing $S$ in its interior. Since $s_{2}(z)$ is schlicht, it omits in $S_{1}$ at least two values, so by Lindelöf's theorem [4, p. 260] we have $\lim _{z \rightarrow 1} s_{2}(z)=L$ where the limit is approached uniformly as $|z| \rightarrow 1$ for $z \in S$. Therefore, since $0<|L|<\infty$, the inequality

$$
\left|s_{2}(z) / s_{2}\left(r_{n}\right)-1\right| \leqq\left|s_{2}\left(r_{n}\right)\right|^{-1}\left\{\left|s_{2}(s)-L\right|+\left|L-s_{2}\left(r_{n}\right)\right|\right\}
$$

shows that $s_{2}(z) / s_{2}\left(r_{n}\right) \rightarrow 1$ as $n \rightarrow \infty$, uniformly for $z \in D_{n}$. This proves the lemma. 
We are now able to determine the asymptotic behavior of $a_{n}$ when $\omega>0$.

THEOREM 3.3. Let $f(z)=z+a_{2} z^{2}+a_{3} z^{3}+\cdots \in V_{k}$. Let

$$
\omega=\lim _{r \rightarrow 1}(1-r)^{k / 2+1}\left|f^{\prime}\left(r \exp \left(i \theta_{0}\right)\right)\right|>0 .
$$

Then if $\rho_{n}=1-1 / n$, we have as $n \rightarrow \infty$

$$
a_{n} \sim \frac{f^{\prime}\left(\rho_{n} \exp \left(i \theta_{0}\right)\right)}{n^{2} \Gamma(k / 2+1)} \exp \left(-i(n-1) \theta_{0}\right)
$$

Proof. Since $\omega>0$ we have

$$
f^{\prime}(z)=\frac{1}{\left(1-z \exp \left(-i \theta_{0}\right)\right)^{k / 2+1}}\left\{\frac{z}{s_{2}(z)}\right\}^{(k-2) / 4} .
$$

Let $\omega(r)$ be as before, and let $\omega_{n}=\omega\left(\rho_{n}\right)$. Let

Then

$$
f_{n}^{\prime}=\frac{\omega_{n}}{\left(1-z \exp \left(-i \theta_{0}\right)\right)^{k / 2+1}}=\omega_{n} \sum_{m=0}^{\infty} C_{m} \exp \left(-i m \theta_{0}\right) z^{m}
$$

$$
C_{m}=\frac{\Gamma(m+k / 2+1)}{\Gamma(m+1) \Gamma(k / 2+1)} \sim \frac{m^{k / 2}}{\Gamma(k / 2+1)}
$$

Straightforward computation shows that

$$
\begin{aligned}
& n a_{n}-\omega_{n} C_{n-1} \exp \left(-i(n-1) \theta_{0}\right) \\
& =\frac{1}{2 \pi \rho^{n-1}} \int_{-\pi}^{\pi}\left\{f^{\prime}\left(\rho e^{i \theta}\right)-f_{n}^{\prime}\left(\rho e^{i \theta}\right)\right\} e^{-i(n-1) \theta} d \theta
\end{aligned}
$$

Let $\delta>0$ be given and choose $C(\delta)$ as in Lemma 3.1. Then by Lemma 3.1, there exists $n_{0}(\delta)$ such that $n \geqq n_{0}(\delta)$ implies $(1 / 2 \pi) \int_{\gamma_{n}}\left|f^{\prime}\left(\rho_{n} e^{i \theta}\right)\right| d \theta<\delta n^{k / 2}$ where $\gamma_{n}$ $=\left\{\theta:\left(1-\rho_{n}\right) C(\delta) \leqq\left|\theta-\theta_{0}\right| \leqq \pi\right\}$. Also, using the fact that $\left|\omega_{n}\right| \rightarrow \omega<\infty$, we see that the conclusion of Lemma 3.1 holds for $f_{n}^{\prime}(z)$ also. Thus

$$
\left|\frac{1}{2 \pi} \int_{y_{n}}\left\{f^{\prime}\left(\rho_{n} e^{i \theta}\right)-f_{n}^{\prime}\left(\rho_{n} e^{i \theta}\right)\right\} e^{-i(n-1) \theta} d \theta\right|<\dot{2 \delta n^{k / 2}} \text { for } n \geqq n_{0}(\delta) .
$$

Let $\gamma_{n}^{\prime}=[-\pi, \pi]-\gamma_{n}=\left\{\theta: 0 \leqq\left|\theta-\theta_{0}\right| \leqq\left(1-\rho_{n}\right) C(\delta)\right\}$. Since $\theta \in \gamma_{n}^{\prime}$ implies $\left|\arg \left(1-\rho_{n} \exp i\left(\theta-\theta_{0}\right)\right)\right| \leqq \tan ^{-1}\left(\left|\theta-\theta_{0}\right| /\left(1-\rho_{n}\right)\right) \leqq \tan ^{-1} C(\delta)<\pi / 2$, we may choose a Stolz angle $S$ (depending on $\delta$ ) with vertex $\exp \left(i \theta_{0}\right)$ such that $\left\{z=\rho_{n} e^{i \theta}: \theta \in \gamma_{n}^{\prime}\right\} \subset S$ for large $n$. Then by Lemma 3.2, $f_{n}^{\prime}\left(\rho_{n} e^{i \theta}\right) \sim f^{\prime}\left(\rho_{n} e^{i \theta}\right)$ as $n \rightarrow \infty$, uniformly for $\theta \in \gamma_{n}^{\prime}$. Therefore $f^{\prime}\left(\rho_{n} e^{i \theta}\right)-f_{n}^{\prime}\left(\rho_{n} e^{i \theta}\right)=o\left\{f_{n}^{\prime}\left(\rho_{n} e^{i \theta}\right)\right\}$ as $n \rightarrow \infty$, where the term $o$ is uniform for $\theta \in \gamma_{n}^{\prime}$. Since $\left|\omega_{n}\right| \rightarrow \omega<\infty,\left|f_{n}^{\prime}\left(\rho e^{i \theta}\right)\right|=O(1-\rho)^{-(k / 2+1)}$, so $f^{\prime}\left(\rho_{n} e^{i \theta}\right)-f_{n}^{\prime}\left(\rho_{n} e^{i \theta}\right)=o\left\{n^{k / 2+1}\right\}$ as $n \rightarrow \infty$, uniformly for $\theta \in \gamma_{n}^{\prime}$. Therefore

$$
\left|\int_{\gamma_{n}^{\prime}}\left\{f^{\prime}\left(\rho_{n} e^{i \theta}\right)-f_{n}^{\prime}\left(\rho_{n} e^{i \theta}\right)\right\} e^{-i(n-1) \theta} d \theta\right| \leqq 2 C(\delta)\left(1-\rho_{n}\right) \cdot o\left(n^{k / 2+1}\right)=o\left(n^{k / 2}\right)
$$


as $n \rightarrow \infty$. Note that although the first $o$ depends upon $\delta$, once we fix $\delta$ and thus $C(\delta)$, we have that $o$ approaches zero independently of $\delta$, so its product with $C(\delta)$ also approaches zero as $n \rightarrow \infty$.

Since $\gamma_{n} \cup \gamma_{n}^{\prime}=[-\pi, \pi]$, by combining (3.4), (3.5), and (3.6) we see

$$
\rho_{n}^{n-1}\left|n a_{n}-\omega_{n} C_{n-1} \exp \left(-i(n-1) \theta_{0}\right)\right|<\{2 \delta+o(1)\} n^{k / 2}
$$

for large $n$. But $\rho_{n}^{n-1} \rightarrow e$ as $n \rightarrow \infty$. Since $\delta>0$ was arbitrary and since $o(1)$ approaches zero independently of $\delta$ as explained above, we have

$$
a_{n}=\omega_{n} \frac{C_{n-1}}{n} \exp \left(-i(n-1) \theta_{0}\right)+o\left(n^{k / 2-1}\right) .
$$

Since $C_{n-1} \sim n^{k / 2} / \Gamma(k / 2+1)$ as $n \rightarrow \infty$, (3.8) shows

$$
a_{n} \sim \frac{\omega_{n} \exp \left(-i(n-1) \theta_{0}\right)}{\Gamma(k / 2+1)} n^{k / 2-1}=\frac{f^{\prime}\left(\rho_{n} \exp \left(i \theta_{0}\right)\right) \exp \left(-i(n-1) \theta_{0}\right)}{n^{2} \Gamma(k / 2+1)} .
$$

This completes the proof of the theorem.

We now remove the restriction that $\omega>0$.

THEOREM 3.4. Let $f(z)=z+a_{2} z^{2}+a_{3} z^{3}+\cdots \in V_{k}$. Let

$$
\omega=\lim _{r \rightarrow 1}(1-r)^{k / 2+1} M\left(r, f^{\prime}\right) .
$$

Then $\lim _{r \rightarrow \infty}\left|a_{n}\right| / n^{k / 2-1}=\omega / \Gamma(k / 2+1)$.

Proof. If $\omega>0$, Theorem 3.4 follows from (3.9). Suppose then that $\omega=0$, so $M\left(r, f^{\prime}\right)=o(1-r)^{-k / 2-1}$. From the equation $f(z)=\int_{0}^{z} f^{\prime}(t) d t$, where we integrate along the radius from 0 to $z$, it follows that $\lim _{r \rightarrow 1} \sup (1-r)^{k / 2} M(r, f)<\varepsilon$ for any $\varepsilon>0$. Thus $M(r, f)=o(1-r)^{-k / 2}$. By Theorem 3.5 of [1] we have $\int_{0}^{2 \pi} r\left|f^{\prime}\left(r e^{i \theta}\right)\right| d \theta$ $\leqq B(k) M(r, f)$ where $B(k)$ depends on $k$ alone. Thus

$$
\int_{0}^{2 \pi} r\left|f^{\prime}\left(r e^{i \theta}\right)\right| d \theta=o(1-r)^{-k / 2}
$$

When combined with the standard inequality [3, p. 11] relating coefficients and integral means, this result implies $a_{n}=o\left(n^{k / 2-1}\right)$. This completes the proof of the theorem.

Corollary 3.5. Let $f(z)=z+a_{2} z^{2}+a_{3} z^{3}+\cdots \in V_{k}$ and let $F(z)$ be as in (1.3). Then there exists a positive integer $n_{0}(f)$ depending on $f(z)$ such that $n \geqq n_{0}(f)$ implies $\left|a_{n}\right| \leqq\left|A_{n}\right|$. Equality can hold for infinitely many $n$ if and only if $f(z)$ $=e^{-i \theta} F\left(e^{i \theta} z\right)$ for some $\theta$.

Proof. Simple calculations show that as $n \rightarrow \infty$

$$
A_{n} \sim \frac{2^{k / 2}}{k \Gamma(k / 2)} n^{k / 2-1}
$$


Let $\omega=\lim _{r \rightarrow 1}(1-r)^{k / 2+1} M\left(r, f^{\prime}\right)$. If $\omega=0$, Theorem 3.4 implies $a_{n}=o\left(n^{k / 2-1}\right)$, so clearly Corollary 3.5 holds.

Suppose now that $\omega>0$, and choose $\theta_{0}$ such that

$$
\omega=\lim _{r \rightarrow 1}(1-r)^{k / 2+1}\left|f^{\prime}\left(r \exp \left(i \theta_{0}\right)\right)\right| \text {. }
$$

From the representation formula (1.1) we see that

$$
\omega=\lim _{r \rightarrow 1} \exp \left\{\frac{1}{\pi} \int_{0}^{2 \pi} \log \left|1-r \exp \left(i\left(\theta_{0}-t\right)\right)\right| d \sigma(t)\right\}
$$

where $\mu(t)=\nu(t)-\sigma(t)$ is the normalized integrator for $f(z)$. Simple calculations now show that $\omega \leqq 2^{k / 2-1}$ with equality if and only if $\sigma(t)$ is a step function with single jump $(k / 2-1) \pi$ at $t=\theta_{0}+\pi$. Since we already know what $\nu(t)$ is from Theorem 3.1, it is clear that $\omega=2^{k / 2-1}$ if and only if $f(z)=e^{-i \theta} F\left(e^{i \theta} z\right)$ for some $\theta$.

Thus, if $\omega=2^{k / 2-1}$ we have $\left|a_{n}\right|=\left|A_{n}\right|$ for all $n$. If $\omega<2^{k / 2-1}$, we see from Theorem 3.4 that

$$
\left|a_{n}\right| \sim \frac{\omega n^{k / 2-1}}{\Gamma(k / 2+1)}=\frac{2 \omega n^{k / 2-1}}{k \Gamma(k / 2)} .
$$

By combining (3.10), (3.11), and the fact that $\omega<2^{k / 2-1}$, we see that $\left|a_{n}\right|<\left|A_{n}\right|$ for all sufficiently large $n$. This proves the corollary.

Another consequence of Theorem 3.4 is the following.

COROLlaRY 3.6. Let $f(z)=z+a_{2} z^{2}+a_{3} z^{3}+\cdots \in V_{k}$. Let

$$
L(k, f)=\lim _{n \rightarrow \infty} \frac{\left|a_{n}\right|}{n^{k / 2-1}} .
$$

Let $L(k)=\sup \left\{L(k, f): f \in V_{k}\right\}$. Then $L(k) \leqq 2^{k / 2} / k \Gamma(k / 2)$ with equality if and only if $f(z)=e^{-i \theta} F\left(e^{i \theta} z\right)$, where $F(z)$ is given by (1.3). In particular, $L(k) \rightarrow 0$ as $k \rightarrow \infty$, and the rate of convergence $2^{k / 2} / k \Gamma(k / 2)$ is best possible.

Remarks. (1) $L(k, f)$ exists by Theorem 3.4.

(2) Robertson [14] showed that

$$
\lim _{n \rightarrow \infty} \sup \frac{\left|a_{n}\right|}{n^{k / 2-1}} \leqq \frac{k^{2}+k}{16}\left(\frac{4 e}{k+4}\right)^{(k+4) / 2},
$$

so $L(k) \rightarrow 0$ as $k \rightarrow \infty$. Corollary 3.6 improves the rate of convergence.

Proof. From Theorem $3.4, L(k, f)=\omega / \Gamma(k / 2+1)$. Since $\omega \leqq 2^{k / 2-1}$ with equality if and only if $f(z)=e^{-i \theta} F\left(e^{i \theta} z\right)$, the corollary is proved. A lengthy but straightforward computation shows that the above rate of convergence improves the estimate given by Robertson.

From our results so far, as well as from the definition of $\omega$, it is evident that the quantity $\omega$ acts as a dividing line between those functions which have (in some sense) maximal growth (i.e. $\omega>0$ ) and those which do not. As is to be expected, 
we can say more about the coefficients of functions with $\omega>0$ than we can say about the coefficients of an arbitrary $V_{k}$ function. We have

CoRollary 3.7. Let $f(z)=z+a_{2} z^{2}+a_{3} z^{3}+\cdots \in V_{k}$, and let $\mu(t)$ be the normalized integrator for $f(z)$. Suppose $\omega=\lim _{r \rightarrow 1}(1-r)^{k / 2+1}\left|f^{\prime}\left(r \exp \left(i \theta_{0}\right)\right)\right|>0$. Then for each $n$ we may choose a value of $\arg a_{n}$ such that

$$
\lim _{n \rightarrow \infty}\left\{\arg a_{n}+n \theta_{0}\right\}=\mu\left(\theta_{0}\right) .
$$

Also,

$$
\lim _{n \rightarrow \infty} \frac{a_{n+1}}{a_{n}}=\exp \left(-i \theta_{0}\right) .
$$

Thus the radius $\arg z=\theta_{0}$ of maximal growth of $f(z)$ may be determined from the coefficients $a_{n}$.

Proof. From Theorem 3.3 we have as $n \rightarrow \infty$

$$
n^{2} a_{n}=\left\{\frac{f^{\prime}\left(\rho_{n} \exp \left(i \theta_{0}\right)\right)}{\Gamma(k / 2+1)} \exp \left(-i(n-1) \theta_{0}\right)\right\}\{1+o(1)\}
$$

where $\rho_{n}=1-1 / n$. Thus

$$
\arg a_{n}=\arg f^{\prime}\left(\rho_{n} \exp \left(i \theta_{0}\right)\right)-(n-1) \theta_{0}+o(1) .
$$

In $[9$, p. 12] it is shown that

$$
\lim _{n \rightarrow \infty} \arg f^{\prime}\left(\rho_{n} \exp \left(i \theta_{0}\right)\right)+\theta_{0}=\mu\left(\theta_{0}\right)
$$

where the existence of the limit is part of the conclusion. (See also the proof of Lemma 1 in [12].) By combining (3.15) and (3.16) we arrive at (3.12). Also, (3.13) follows directly from (3.14).

4. Asymptotic formulas for the integral means. In this section we shall use techniques similar to those of $\S 3$ to study the asymptotic behavior of $I_{\lambda}\left(r, f^{\prime}\right)$ and $I_{\lambda}(r, f)$. In [5] it was shown that, with $\lambda \geqq 1$,

$$
I_{\lambda}\left(r, f^{\prime}\right) \leqq\left(\frac{1}{1-r^{2}}\right)^{\lambda}\left(\frac{1+r}{1-r}\right)^{\lambda k / 2-1} .
$$

In [1] it was shown that for any real $\lambda$ with $\lambda(k / 2+1)>1$, we have

$$
\lim _{r \rightarrow 1} \sup (1-r)^{\lambda(k / 2+1)-1} I_{\lambda}\left(r, f^{\prime}\right) \leqq A(k, \lambda) .
$$

The exact value of the constant $A(k, \lambda)$ was given in [1], and it was shown that $A(k, \lambda)$ cannot be improved over the whole class $V_{k}$. We shall show that if $\lambda(k / 2+1)>1$, then

$$
\lim _{r \rightarrow 1}(1-r)^{\lambda(k / 2+1)-1} I_{\lambda}\left(r, f^{\prime}\right)=A(\omega, k, \lambda)
$$


where

$$
A(\omega, \lambda, k)=\frac{\omega^{\lambda} \Gamma(\lambda(k / 2+1)-1)}{2^{\lambda(k / 2+1)-1} \Gamma^{2}(\lambda(k / 2+1) / 2)}
$$

It is interesting to note that here again the quantity $\omega$ plays the role of a dividing line between those functions for which $I_{\lambda}\left(r, f^{\prime}\right)$ has maximal growth and those for which it does not.

We first need two technical lemmas.

LEMMA 4.1. Let $f(z) \in V_{k}$ and let $\omega=\lim _{r \rightarrow 1}(1-r)^{k / 2+1}\left|f^{\prime}\left(r \exp \left(i \theta_{0}\right)\right)\right|>0$. Let $\omega(R)=(1-R)^{k / 2+1}\left|f^{\prime}\left(R \exp \left(i \theta_{0}\right)\right)\right|$. Let $C>0$ and $\lambda>0$ be fixed, and let $\gamma_{R}=\left\{\theta:(1-R) C \leqq\left|\theta-\theta_{0}\right| \leqq \pi\right\}, \gamma_{R}^{\prime}=[-\pi, \pi]-\gamma_{R}$. Let

$$
f_{R}^{\prime}(z)=\frac{\omega(R)}{\left(1-z \exp \left(-i \theta_{0}\right)\right)^{k / 2+1}}
$$

Then

$$
\int_{\gamma_{R}^{\prime}}\left|f_{R}^{\prime}\left(R e^{i \theta}\right)\right|^{\lambda} d \theta \sim \int_{\gamma_{R}^{\prime}}\left|f^{\prime}\left(R e^{i \theta}\right)\right|^{\lambda} d \theta
$$

as $R \rightarrow 1$.

Proof. Throughout the proof we let $z=|z| e^{i \theta}$. Then exactly as in the proof of Lemma 3.2 we see that given a Stolz angle $S$ with vertex at $\exp \left(i \theta_{0}\right)$, we have $f_{R}^{\prime}(z) \sim f^{\prime}(z)$ as $R \rightarrow 1$, uniformly for $|z| \geqq R$ and $\theta \in \gamma_{|z|}^{\prime}$. It follows that $f^{\prime}(z) / f_{R}^{\prime}(z)=1+\varepsilon_{R}(z)$ as $R \rightarrow 1$, where $\varepsilon_{R}(z) \rightarrow 0$ as $R \rightarrow 1$, uniformly for $|z| \geqq R$, $\theta \in \gamma_{|z|}^{\prime}$. Expanding $\left(1+\varepsilon_{R}(z)\right)^{\lambda}$ in powers of $\varepsilon_{R}(z)$, we find

so that

$$
\left|\frac{f^{\prime}(z)}{f_{R}^{\prime}(z)}\right|^{\lambda}=\left|1+\varepsilon_{R}(z)\right|^{\lambda}=1+o(1),
$$

$$
\left|f^{\prime}(z)\right|^{\lambda}-\left|f_{R}^{\prime}(z)\right|^{\lambda}=o\left\{\left|f_{R}^{\prime}(z)\right|^{\lambda}\right\},
$$

where again the term $o$ is uniform as $R \rightarrow 1$ for $|z| \geqq R, \theta \in \gamma_{|z|}^{\prime}$.

Since this term $o$ is uniform in $z$ as stated above, we may integrate and find

$$
\int_{\gamma_{R}^{\prime}}\left|f^{\prime}\left(R e^{i \theta}\right)\right|^{\lambda} d \theta-\int_{\gamma_{R}^{\prime}}\left|f_{R}^{\prime}\left(R e^{i \theta}\right)\right|^{\lambda} d \theta=o\left\{\int_{\gamma_{R}^{\prime}}\left|f_{R}^{\prime}\left(R e^{i \theta}\right)\right|^{\lambda} d \theta\right\}
$$

where again the term $o$ is uniform for $z$ as above. This proves the lemma.

LEMMA 4.2. Let $f(z) \in V_{k}$. Let $\omega, \omega(R)$, and $f_{R}(z)$ be as in Lemma 4.1. Then if $\lambda(k / 2+1)>1$, we have

$$
I_{\lambda}\left(r, f^{\prime}\right)=(\omega(r) / \omega)^{\lambda} I_{\lambda}\left(r, \omega\left(1-z \exp \left(-i \theta_{0}\right)\right)^{-(k / 2+1)}\right)+o\left\{(1-r)^{-\lambda(k / 2+1)+1}\right\}
$$

where the term $o$ is uniform as $r \rightarrow 1$. 
Proof. By the definition of $I_{\lambda}\left(r, f^{\prime}\right)$ we have

$$
\begin{aligned}
2 \pi\left|I_{\lambda}\left(r, f^{\prime}\right)-I_{\lambda}\left(r, f_{R}^{\prime}\right)\right| \leqq & \int_{\gamma}\left|f^{\prime}(z)\right|^{\lambda} d \theta+\int_{\gamma}\left|f_{R}^{\prime}(z)\right|^{\lambda} d \theta \\
& +\left|\int_{\gamma^{\prime}}\left\{\left|f^{\prime}(z)\right|^{\lambda}-\left|f_{R}^{\prime}(z)\right|^{\lambda}\right\} d \theta\right|
\end{aligned}
$$

where $\gamma$ and $\gamma^{\prime}$ are any two disjoint arcs with $\gamma \cup \gamma^{\prime}=[-\pi, \pi]$. Just as in Lemma 3.1, we may choose $C=C(\delta, \lambda)$ and $r_{0}=r_{0}(\delta, \lambda)$ such that $r \geqq r_{0}$ implies

$$
\int_{(1-r) C}^{\pi}\left|f^{\prime}\left(r e^{i \theta}\right)\right|^{\lambda} d \theta<\frac{\delta}{(1-r)^{\lambda(k / 2+1)-1}}
$$

(In the proof of $(4.4)$ it is essential that $\lambda(k / 2+1)>1$.) The same result clearly holds for $f_{R}^{\prime}(z)$. Let $\gamma=\left\{r e^{i \theta}:(1-r) C \leqq\left|\theta-\theta_{0}\right| \leqq \pi\right\}$, and let $\gamma^{\prime}=[-\pi, \pi]-\gamma$. Then from (4.3), (4.4), and Lemma 4.1 we see that, as $r \rightarrow 1$,

$$
2 \pi\left|I_{\lambda}\left(r, f^{\prime}\right)-I_{\lambda}\left(r, f_{r}^{\prime}\right)\right|<\left\{2 \delta+\omega(r)^{\lambda} C(\delta, \lambda) o(1)\right\}(1-r)^{-\lambda(k / 2+1)+1} .
$$

As explained in the proof of Lemma 3.1, this implies

$$
I_{\lambda}\left(r, f^{\prime}\right)-I_{\lambda}\left(r, f_{r}^{\prime}\right)=o(1-r)^{-\lambda(k / 2+1)+1}
$$

as $r \rightarrow 1$, which proves Lemma 4.2.

We are now able to establish (4.1) when $\omega>0$.

THEOREM 4.1. Let $f(z) \in V_{k}$ with $\omega>0$. Let $\lambda(k / 2+1)>1$. Then

$$
\lim _{r \rightarrow 1}(1-r)^{\lambda(k / 2+1)-1} I_{\lambda}\left(r, f^{\prime}\right)=A(\omega, \lambda, k)
$$

where $A(\omega, \lambda, k)$ is given by (4.2).

Proof. Without loss of generality we assume $\theta_{0}=0$. In [11] Pommerenke showed that

$$
\frac{1}{2 \pi} \int_{-\pi}^{\pi} \frac{1}{\left|1+r e^{i \theta}\right|^{m}} d \theta \sim \frac{\Gamma(m-1)}{2^{m-1} \Gamma^{2}(m / 2)} \frac{1}{(1-r)^{m-1}}
$$

as $r \rightarrow 1$, whenever $m>1$. Let $m=\lambda(k / 2+1)$. Combining (4.5) with Lemma 4.2, we obtain (4.1). This proves the theorem.

We now establish (4.1) when $\omega=0$.

THEOREM 4.2. Let $f(z) \in V_{k}$ with $\omega=0$. Let $\lambda(k / 2+1)>1$. Then

$$
\lim _{r \rightarrow 1}(1-r)^{\lambda(k / 2+1)-1} I_{\lambda}\left(r, f^{\prime}\right)=0 .
$$

Proof. There exist starlike functions $s_{1}(z)$ and $s_{2}(z)$ such that

$$
f^{\prime}(z)=\frac{\left(s_{1}(z) / z\right)^{(k+2) / 4}}{\left(s_{2}(z) / z\right)^{(k-2) / 4}}
$$


Suppose $s_{1}(z)=z /\left(1-e^{i \theta} z\right)^{2}$. Without loss of generality we assume $\theta=0$. Then just as in the proof of Lemma 3.1, we see that given $\delta>0$ we may choose $C=C(\delta)$ and $r_{0}=r_{0}(\delta)$ such that $r \geqq r_{0}(\delta)$ implies

$$
\int_{(1-r) C}^{\pi}\left|f^{\prime}\left(r e^{i \theta}\right)\right|^{\lambda} d \theta<\delta(1-r)^{-\lambda(k / 2+1)+1}
$$

(Again $\lambda(k / 2+1)>1$ is essential.) Since $\omega=0, M\left(r, f^{\prime}\right)=\varepsilon(r)(1-r)^{-k / 2-1}$ where $\varepsilon(r) \rightarrow 0$ as $r \rightarrow 1$. Thus

$$
\int_{0}^{(1-r) C}\left|f^{\prime}\left(r e^{i \theta}\right)\right|^{\lambda} d \theta \leqq \frac{\varepsilon(r) C(\delta)}{(1-r)^{\lambda(k / 2+1)-1}}
$$

These two facts imply

$$
\int_{0}^{\pi}\left|f^{\prime}\left(r e^{i \theta}\right)\right|^{\lambda} d \theta=o\left\{(1-r)^{-\lambda(k / 2+1)+1}\right\} .
$$

Since the same argument is valid on $[-\pi, 0]$, the proof is complete.

Suppose now that $s_{1}(z) \neq z /\left(1-e^{i \theta} z\right)^{2}$. By Theorem 1 of [12] (or directly from (1.1)), we see that there exists $\beta<2$ and a constant $A(\beta)$ such that $\left|s_{1}(z)\right|$ $\leqq A(\beta) /(1-r)^{\beta}$. We may clearly choose $\beta$ such that $\lambda \beta((k+2) / 4) \neq 1$. By noting that $s_{1}(z)$ is schlicht, we may use Theorem 3.2 in [3, p. 45]. We see that given $r_{0}<1$

$$
(1-r)^{\lambda(k / 2+1)-1} I_{\lambda}\left(r, f^{\prime}\right) \leqq A\left(r_{0}\right)(1-r)^{\lambda(k / 2+1)-1}+B\left(r_{0}\right)(1-r)^{\delta}
$$

for $r \geqq r_{0}$, where $\delta=\lambda(2-\beta)(k+2) / 4>0$. We now let $r \rightarrow 1$ to complete the proof of the theorem.

Theorems 4.1 and 4.2 improve the results in [1] in the sense that we can say that the limit in (4.1) exists. Also, since $\omega \leqq 2^{k \beta-1}$ with equality if and only if $f(z)$ $=e^{-i \theta} F\left(e^{i \theta} z\right)$ where $F(z)$ is given by (1.3), we can state the conditions under which equality may hold in the result in [1].

After examining these results on $I_{\lambda}\left(r, f^{\prime}\right)$, it might seem natural to expect that corresponding results would hold for $I_{\lambda}(r, f)$. We have not been able to show this, but we do have the following theorem.

THEOREM 4.3. Let $f(z) \in V_{k}$ and let $\lambda k / 2>1$. Then

$$
\lim _{r \rightarrow 1} \sup (1-r)^{\lambda k / 2-1} I_{\lambda}(r, f) \leqq \frac{\lambda k / 2}{\lambda k / 2-1}\left(\frac{2 \omega}{k}\right)^{\lambda} .
$$

In particular, if $\omega=0$, then the limit exists and is zero.

Proof. By a result of Brannan [1], $f(z)$ is at most $k / 2$ valent. The Hardy-SpencerStein identities [3, p. 42] then show that for any fixed $r_{0}<1$ and $r \geqq r_{0}$

$$
I_{\lambda}(r, f)-I_{\lambda}\left(r_{0}, f\right) \leqq \lambda k / 2 \int_{r_{0}}^{r} \frac{M(t, f)}{t} d t .
$$


By combining the definition of $\omega$ with the inequality $M(r, f) \leqq \int_{0}^{r} M\left(t, f^{\prime}\right) d t$, we see that

$$
\lim _{r \rightarrow 1} \sup (1-r)^{k / 2} M(r, f) \leqq 2 \omega / k .
$$

By combining (4.7) with (4.6), we see that given $\varepsilon>0$ there exists $r(\varepsilon)<1$ such that $r \geqq r(\varepsilon)$ implies

$$
I_{\lambda}(r, f) \leqq I_{\lambda}(r(\varepsilon), f)+\frac{1}{r(\varepsilon)} \frac{\lambda k / 2}{\lambda k / 2-1}\left(\frac{2 \omega}{k}+\varepsilon\right)^{\lambda} \frac{1}{(1-r)^{\lambda k / 2-1}} .
$$

By letting $r \rightarrow 1$, we see that

$$
\lim _{r \rightarrow 1} \sup (1-r)^{\lambda k / 2-1} I_{\lambda}(r, f) \leqq \frac{1}{r(\varepsilon)} \frac{\lambda k / 2}{\lambda k / 2-1}\left(\frac{2 \omega}{k}+\varepsilon\right)^{\lambda} .
$$

This in turn implies the theorem.

We now turn our attention to $\lim _{r \rightarrow 1} \inf (1-r)^{\lambda k / 2-1} I_{\lambda}(r, f)$. We first need a technical lemma.

LEMMA 4.3. Let $f(z)$ be analytic in $U$ and suppose $f^{\prime}(z) \neq 0$ in $U$. Then for $\lambda>0$ we have

$$
\int_{0}^{2 \pi}\left|f\left(r e^{i \theta}+\frac{1-r}{2} e^{i x}\right)\right|^{\lambda} d \theta \leqq \frac{1}{r} \int_{0}^{2 \pi}\left|f\left(\left(r+\frac{1-r}{2}\right) e^{i \theta}\right)\right|^{\lambda} d \theta
$$

for all $x \in[0,2 \pi]$ and for all $r, 0<r<1$.

Proof. Let $x \in[0,2 \pi]$ be given. Fix $r<1$ and let $a=((1-r) / 2) e^{i x}$. Let

$$
g_{r}(z)=f\left(\frac{r+(1-r) / 2}{r} z\right) \text { and } g_{a}(z)=f(z+a) \text {. }
$$

Let $b=r a /(r+(1-r) / 2)$, so $|b|<r$. Let

$$
T(z)=(z+b) /\left(1+\bar{b} r^{-2} z\right) .
$$

Then $T(z)$ maps $U_{r}=\{z:|z|<r\}$ onto $U_{r}$ with $T(0)=b$.

Now $g_{a}(0)=g_{r} \circ T(0)=f(a)$. Also $g_{a}\left(U_{r}\right) \subset g_{r} \circ T\left(U_{r}\right)$. Thus $g_{a}(z) \prec g_{r} \circ T(z)$ in $U_{r}$. (Note that $f^{\prime}(z) \neq 0$ is needed to prove the subordination.) Therefore

$$
\int_{0}^{2 \pi}\left|f\left(r e^{i \theta}+a\right)\right|^{\lambda} d \theta \leqq \int_{0}^{2 \pi}\left|g_{r} \circ T\left(r e^{i \theta}\right)\right|^{\lambda} d \theta .
$$

Since $T(z)$ maps $|z|=r$ onto itself, let $T\left(r e^{i \theta}\right)=r e^{i \Phi}$. Then on $|z|=r$ we have

$$
r d \Phi=4 r^{2} /\left|1+r+(1-r) e^{i(\theta-x)}\right|^{2} d \theta .
$$

Combining this with (4.8) we see that

$$
\int_{0}^{2 \pi}\left|f\left(r e^{i \theta}+a\right)\right|^{\lambda} d \theta \leqq \frac{1}{r} \int_{0}^{2 \pi}\left|g_{r}\left(r e^{i \Phi}\right)\right|^{\lambda} d \Phi
$$

which is equivalent to the conclusion of the lemma. 
We now prove

THEOREM 4.4. Let $f(z) \in V_{k}$ and let $\lambda \geqq 1$. Then

$$
\lim _{r \rightarrow 1} \inf (1-r)^{\lambda k / 2-1} I_{\lambda}(r, f) \geqq \frac{A(\omega, \lambda, k)}{2^{\lambda(k / 2+1)-1}}
$$

where $A(\omega, \lambda, k)$ is given by (4.2).

Proof. Since $\lambda \geqq 1$ we have from a generalization of Minkowski's inequality $[15$, vol. I, p. 260] that

$$
I_{\lambda}\left(r, f^{\prime}\right)^{1 / \lambda} \leqq \frac{1}{\pi(1-r)} \int_{0}^{2 \pi}\left\{\frac{1}{2 \pi} \int_{0}^{2 \pi}\left|f\left(r e^{i \theta}+(\delta / 2) e^{i x}\right)\right|^{\lambda} d \theta\right\}^{1 / \lambda} d x
$$

where $\delta=1-r$. From Theorems 4.1 and 4.2 we have

$$
I_{\lambda}\left(r, f^{\prime}\right)^{1 / \lambda}=\left\{\frac{A(\omega, \lambda, k)+o(1)}{(1-r)^{\lambda(k / 2+1)-1}}\right\}^{1 / \lambda} .
$$

By combining (4.9), (4.10), and Lemma 4.3 we see that

$$
\left\{\frac{A(\omega, \lambda, k)+o(1)}{(1-r)^{\lambda k / 2-1}}\right\}^{1 / \lambda} \leqq \frac{2}{r^{1 / \lambda}}\left\{\frac{1}{2 \pi} \int_{0}^{2 \pi}\left|f\left((r+(1-r) / 2) e^{i \theta}\right)\right|^{\lambda} d \theta\right\}^{1 / \lambda}
$$

We now let $(1+r) / 2=t$, so $(1-r)=2(1-t)$. Substituting this in (4.11) and letting $t \rightarrow 1$, we arrive at the conclusion of the theorem.

Corollary 4.5. Let $f(z) \in V_{k}$. Let $\lambda$ satisfy $\lambda \geqq 1$ and $\lambda k / 2>1$. Then

$$
\lim _{r \rightarrow 1} \sup (1-r)^{\lambda k / 2-1} I_{\lambda}(r, f)=0
$$

if and only if

$$
\lim _{r \rightarrow 1} \inf (1-r)^{\lambda k / 2-1} I_{\lambda}(r, f)=0 .
$$

Proof. Clearly if the lim sup is 0 , so is the lim inf. The reverse implication is proved by Theorems 4.3 and 4.4 .

Although we have established the existence of $\lim _{r \rightarrow 1}(1-r)^{\lambda k / 2-1} I_{\lambda}(r, f)$ only when $\omega=0$, Corollary 4.5 tells us that the growth of $I_{\lambda}(r, f)$ is quite regular.

\section{BIBLIOGRAPHY}

1. D. A. Brannan, On functions of bounded boundary rotation. I, Proc. Edinburgh Math. Soc. $16(1968 / 69), 339-347$.

2. - On functions of bounded boundary rotation. II, Bull. London Math. Soc. 1 (1969), 321-322.

3. W. K. Hayman, Multivalent functions, Cambridge Tracts in Math. and Math. Phys., no. 48, Cambridge Univ. Press, Cambridge, New York, 1958. MR 21 \#7302.

4. E. Hille, Analytic function theory. Vol. II, Introduction to Higher Math., Ginn, Boston, Mass., 1962. MR 34 \#1490.

5. W. E. Kirwan, On the coefficients of functions with bounded boundary rotation, Michigan Math. J. 15 (1968), 277-282. MR 38 \#1250. 
6. $\mathrm{O}$. Lehto, On the distortion of conformal mappings with bounded boundary rotation, Ann. Acad. Sci. Fenn. Ser. A I Math.-Phys. No. 124 (1952). MR 14, 743.

7. J. E. Littlewood, Lectures on the theory of functions, Oxford Univ. Press, Oxford, 1944. MR 6, 261.

8. K. Löwner, Untersuchungen über die Verzerrung bei konformen Abbildungen des Einheitskreises $|z|<1$, die durch Funktionen mit nicht verschwindender Ableitung geliefert werden, Leipzig Berichte 69 (1917), 89-106.

9. J. W. Noonan, Asymptotic expressions for functions of bounded boundary rotation, Ph.D. Thesis, University of Maryland, College Park, Md., 1971.

10. V. Paatero, Uber die konforme Abbildung von Gebieten deren Rander von beschrankter Drehung sind, Ann. Acad. Sci. Fenn. Ser. A I Math.-Phys. No. 33 (1931).

11. Ch. Pommerenke, On the coefficients of close-to-convex functions, Michigan Math. J. 9 (1962), 259-269. MR 26 \#5153.

12. - On starlike and convex functions, J. London Math. Soc. 37 (1962), 209-224. MR 25 \#1279.

13. A. Rényi, On the coefficients of schlicht functions, Publ. Math. Debrecen 1 (1949), 18-23. MR 11, 92.

14. M. S. Robertson, Coefficients of functions with bounded boundary rotation, Canad. J. Math. 21 (1969), 1477-1482. MR 41 \#458.

15. A. Zygmund, Trigonometrical series, 2nd rev. ed., vols. I, II, Cambridge Univ. Press, New York, 1959. MR 21 \#6498.

E. O. Hulburt Center for Space Research, Naval Research laboratory, Washington, D. C. 20390

Current address: Department of Mathematics, College of the Holy Cross, Worcester, Massachusetts 01610 